\title{
China y América Latina: liderazgo organizacional intercultural en la búsqueda de la "comunidad de destino compartido"
}

China and Latin America: intercultural organizational leadership in the search for "community of shared destiny"

Esteban Zottele ${ }^{1}$

\section{Resumen:}

La globalización ha sido una constante en los últimos 30 años. La cooperación entre gobiernos, los contactos de empresas y de diversas instituciones han generado un marcado crecimiento en el intercambio de personas entre países; sin embargo, estos procesos que han profundizado vínculos económicos y comerciales no siempre han tenido en cuenta aspectos fundamentales de las culturas nacionales y sus individuos.

La Franja y la Ruta propone una "comunidad de destino compartido", con la consecuente necesidad de mayor entendimiento mutuo que de esta consigna se deriva. La diversidad cultural plantea importantes problemas para el diseño y la dirección de las organizaciones transnacionales. En estos casos es fundamental generar un liderazgo organizacional intercultural, que puedan aportar soluciones a los nuevos desafíos, logrando efectividad en los resultados planificados.

Este trabajo tratará de comprender esta situación a través del análisis de dimensiones culturales y liderazgo para aportar nuevas direcciones acerca de la importancia del liderazgo intercultural ante la iniciativa de La Franja y la Ruta.

Palabras claves: Globalización, La Franja y Ruta, Cultura Nacional, Liderazgo intercultural.

\footnotetext{
${ }^{1}$ Profesor de la Universidad de Changzhou (CCZU), Investigador del Centro de Estudios Latinoamericanos de la Universidad de Changzhou (CELA) y del Centro de Estudios China- Veracruz (Cechiver) de la Universidad Veracruzana (UV). Representante de la Universidad Veracruzana en China, con PhD por la Universidad de Renmin de China.
} 


\begin{abstract}
:
In the past 30 years, globalization has been a continuous process. Intergovernmental cooperation, business and institutional exchanges have led to a significant increase in people to people exchanges between countries, but these processes have deepened economic and trade ties, they do not always take into account the fundamental aspects of national culture and its individuals.
\end{abstract}

The Belt and Road Initiative had set out a "Community of Common Destiny", so there needs to be greater mutual understanding. Cultural diversity poses a major challenge to the design and management of transnational organizations. In this context, it is essential to develop cross-cultural organizational leadership that can provide solutions to new challenges and effectively deliver planned results.

Through an analysis of the cultural dimension and types of leadership, this work will attempt to understand the situation in order to provide new guidance on the importance of cross-cultural leadership to Belt and Road Initiative.

Key words: Globalization, Belt and Road Initiative, National culture, Cross-cultural leadership.

Orientando. Temas de Asia Oriental. Sociedad, Cultura y Economía. Revista editada por el Centro de Estudios China-Veracruz de la Universidad Veracruzana, México (Centro de Estudios APEC) / año 10 / número 19/ 


\section{Introducción}

En las últimas décadas, con la globalización acelerada a través de las integraciones económicas, la cooperación entre gobiernos, las fusiones, adquisiciones y la expansión entre empresas, se ha ido desarrollando un gran intercambio entre países de distintas regiones. Sin embargo, el mismo ha sido generado básicamente por motivaciones económicas, y en muchas ocasiones no ha tenido en cuenta aspectos fundamentales de las culturas nacionales y sus individuos, que constituyen la base del entendimiento mutuo genuino que puede generar cooperaciones a largo plazo entre países en la búsqueda de objetivos comunes.

A partir de 2013, la iniciativa de la Franja y la Ruta ha propuesto un cambio de paradigma en las relaciones internacionales, con nuevos desafíos no solo para China, sino también para todos los países participantes, entre los cuales, la coordinación de políticas, el financiamiento, la conectividad de infraestructuras, el comercio y el intercambio de personas, figuran como algunos de los grandes retos para la implementación. Sin embargo el intercambio de personas actúa como una variable fundamental cuando pensamos en una aplicación efectiva de dicha iniciativa.

El intercambio de personas entre distintos países puede colaborar a acortar la brecha del conocimiento entre la teoría y la práctica, proceso fundamental para la planificación; por tanto, permite tener una noción más efectiva sobre los problemas que se suscitan a lo largo de la implementación de los planes y sobre todo mejorar el entendimiento mutuo, con vista a futuras cooperaciones. No obstante, dicho intercambio entre diferentes culturas es complejo y a menudo se pueden generar malentendidos derivados de las diferencias, no solo culturales, sino también históricas, políticas y jurídicas.

Hofstede (1980) y Triandis (1995) consideran que los valores y las creencias de los miembros de una cultura influyen en la formación del comportamiento individual, colectivo e institucional entre culturas y el grado en que se consideran legítimos, aceptables y eficaces. En este sentido es fundamental el rol del liderazgo efectivo para poder llevar a cabo con éxito los procesos organizacionales cross-nacionales. 
Figura 1. Intercambio de personas en el marco de la iniciativa de La Franja y la Ruta

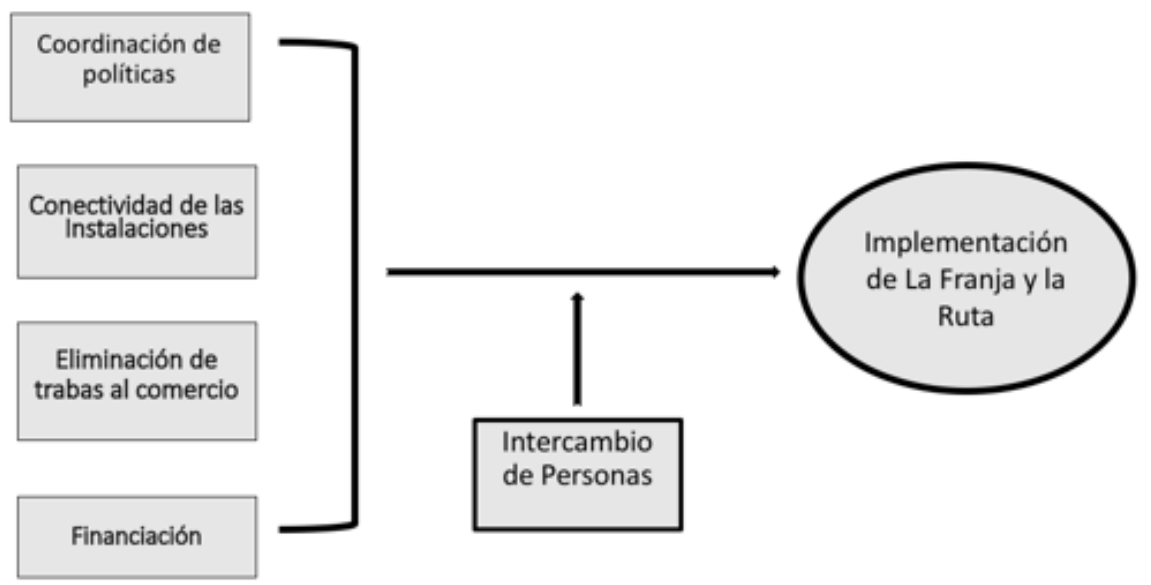

Fuente: Elaboración propia. (Basado en Zottele, 2019)

Un liderazgo Intercultural deberá ser la base activa en la implementación de todo tipo de proyectos, sea a nivel empresarial, educativo, de asociación o entre gobiernos en el marco de la iniciativa.

\section{La Franja y la Ruta y el intercambio de personas}

La iniciativa de La Franja y la Ruta, anunciada en 2013 por el Presidente Xi Jinping, ha sido base fundamental de la política exterior china en los últimos años. Wang Yiwei (2019) afirma que la iniciativa de La Franja y la Ruta tiene su origen en China pero pertenece a todo el mundo.

El sistema económico internacional se ha globalizado, pero la estructura política mundial todavía se basa en Estados nación; esta es la parte más vulnerable de la gobernanza global. En este sentido, el éxito de la Franja y la Ruta dependerá de su capacidad para resolver los problemas más grandes e importantes de la sociedad humana. La Franja y la Ruta, al tratar los problemas del mundo, también implica el actuar respecto a los problemas de China.

Orientando. Temas de Asia Oriental. Sociedad, Cultura y Economía. Revista editada por el Centro de Estudios China-Veracruz de la Universidad Veracruzana, México (Centro de Estudios APEC) / año 10 / número 19/ 
La iniciativa de la Franja y la Ruta se basa en la idea de una "comunidad de destino compartido", por eso el intercambio de personas es la columna vertebral del fomento del conocimiento mutuo. El intercambio de personas se puede producir a través de distintas áreas, podemos destacar las siguientes: intercambio entre empresas, intercambio entre gobiernos, intercambio entre medios de prensa, intercambio entre universidades y organizaciones académicas e intercambio turístico.

\subsection{Intercambio entre empresas}

Además de seguir sosteniendo un incremento en las operaciones de compra y venta de bienes y servicios, deberán incrementarse cooperaciones estratégicas con énfasis en inversiones mutuas y colaboraciones entre empresas de distintos tamaños que produzcan una sinergia hacia el incremento del desarrollo económico entre regiones. Es fundamental el caso de las Asociaciones entre pequeñas y medianas empresas (Pymes) entre los países que pertenecen a la Franja y La Ruta, porque en el caso de América Latina, este tipo de empresas representan más del $90 \%$ del total de las empresas, siendo el gran motor de las economías nacionales.

\subsection{Intercambio entre gobiernos}

El intercambio de funcionarios entre gobiernos será una herramienta vital para poder realizar una efectiva coordinación de políticas; en este caso, el conocimiento de los actores principales podrá contribuir a mejorar la comunicación entre gobiernos, para desarrollar la cooperación en distintas áreas relacionadas con aspectos legales, impuestos, facilitación de aspectos migratorios, monetarios, uso de nuevas tecnologías, etc. Tales elementos facilitarán no solo los intercambios a nivel empresarial y gubernamental, sino que también la vida cotidiana de la población de los países involucrados.

\subsection{Intercambio entre medios de prensa}

En América Latina y China, muchas veces permean las coberturas de agencias internacionales que no permiten el conocimiento directo de la noticia con la interpretación cultural que esta conlleva. El intercambio entre medios de prensa deberá fomentar el contacto directo entre países, pudiendo obtener información generada y difundida entre las partes, lo cual implicará mayor acercamiento e información útil para el incremento de las relaciones entre regiones. 


\subsection{Intercambio entre universidades y organizaciones académicas}

En los últimos años, el intercambio entre universidades de China y América Latina ha incrementado. En este sentido, además de la creación de Centros de Estudios Latinoamericanos en China y de Centros de Estudios sobre Asia en América Latina, el aprendizaje de ambos idiomas también ha crecido de manera exponencial. Sin embargo, debe fomentarse un intercambio más genuino, generando una mejora en la calidad de las investigaciones en conjunto y la comprensión cultural: investigaciones que deberán generar mayor conocimiento para reducir la brecha que existe entre la teoría y la práctica en los estudios entre regiones.

\subsection{Intercambio turístico}

Para facilitar el desarrollo turístico entre regiones, la gestión de este servicio requerirá un alto grado de comprensión intercultural, ya que el turismo genera un genuino intercambio entre las regiones. El turismo se comporta como un elemento dinamizador del patrimonio y las comunidades, impulsa el reconocimiento y la creación de un sentimiento de orgullo comunitario; es factor de divulgación del patrimonio. Como valor para la cultura, genera recursos para la conservación y beneficia a las comunidades receptoras, motiva a las comunidades en la administración de su patrimonio y crea conciencia del valor de los diferentes "patrimonios locales" entre los turistas. (Sectur, 1997 )

\section{Distintos conceptos sobre cultura}

La manera en que los individuos reaccionan ante un fenómeno similar, cómo analizan los hechos y cómo responden a estímulos determinados, es muy diferente entre las distintas culturas nacionales. Las diferencias culturales se manifiestan de diversas maneras. La manifestación más profunda de la cultura es el conjunto de valores. Los valores son tendencias generales a preferir ciertos estados de las cosas más que otros. Las diferentes culturas tienen sus propias pautas acerca de lo que es una conducta aceptable o inaceptable. (Fanjul, 2010)

Orientando. Temas de Asia Oriental. Sociedad, Cultura y Economía. Revista editada por el Centro de Estudios China-Veracruz de la Universidad Veracruzana, México (Centro de Estudios APEC) / año 10 / número 19/ 
Smith (1966) indica que la cultura es un sistema aprendido de conocimientos, comportamientos, actitudes, creencias, valores y normas que es compartido por un grupo de personas. En el sentido más amplio, incluye la forma en que las personas piensan, lo que hacen y cómo utilizan el entorno, resultando de la diversidad la naturaleza única de cada cultura. Los elementos, los valores, y el contexto de cada una se distinguen de todas las demás. (Beebe, Beebe \& Redmond, 2005)

Por otra parte, Bodlye (1994) concibe a la cultura como algo que se transmite de generación en generación a través del aprendizaje; adquirir una cultura, por lo tanto, no significa nacer con ella, sino aprender los significados compartidos presentes en la misma, lo que permite de alguna manera pertenecer a ella y poseer una identidad. En este sentido, la cultura va estrechamente unida con el proceso de socialización primaria, que tiene lugar por medio de la familia, la escuela, la religión y los medios masivos de difusión, entre otras instancias. (Rizo, M., \& Romeu, V. 2006)

Williams (1983) sostiene que la dificultad de definir la cultura desde un planteamiento científico radica en su extensión histórica, geográfica e intelectual. Para definirla, por tanto, debe ser entendida desde la visión de un concepto flexible que se adapta a múltiples circunstancias y objetivos de investigación. (Trujillo Sáez, F. 2005)

A pesar que no existe consenso internacional sobre la definición del término, existe acuerdo entre los especialistas (Triandis, 2000), de que el núcleo de la cultura está constituido por los valores. Los valores son objetivos deseables y trans-situacionales, que orientan conductas y sirven como principios que guían la vida. ( Omar \& Urteaga, 2010)

Para Hofstede no existe la "cultura" en un sentido tangible. Es un producto de nuestra imaginación y solo nos resulta útil en la medida en que nos ayuda a entender y a predecir fenómenos del mundo real. La cultura nacional y la cultura organizacional constituyen dos fenómenos muy diferentes: las culturas nacionales pertenecen al ámbito de la antropología; las organizacionales al de la sociología.

La gestión empresarial nunca podrá modificar una cultura nacional: únicamente podrá entenderla y utilizarla, pero sí puede crear y, en ocasiones, cambiar una cultura organizacional. El concepto de "cultura" no se aplica a los individuos; los individuos tienen personalidades, en las que influye la cultura en la que crecieron solo en una parte. 
Omar, A., \& Urteaga, A. F. 2010) En suma, en el estudio de las organizaciones, resulta fundamental comprender la diferencia y la relación que existe entre cultura organizacional y cultura nacional.

\section{a. Cultura organizacional}

Las organizaciones son unidades sociales de personas que se estructuran y gestionan para satisfacer una necesidad o para perseguir objetivos colectivos. (G. Joseph Sowa 2006) Todas las organizaciones tienen una estructura de gestión, la cual determina las relaciones entre las diferentes actividades y los miembros; esta se subdivide en: roles, responsabilidades y autoridades para llevar a cabo diferentes tareas. Las organizaciones son sistemas abiertos que afectan y son afectados por su entorno (Ludwig von Bertanlanffy, 1956) e intercambian información periódicamente. Los sistemas abiertos tienen insumos, procesos, resultados, objetivos, evaluación y aprendizaje; los límites, el medio ambiente externo y la equidad son aspectos de importancia crítica. ( Zottele, 2015 )

La manera en que las organizaciones se relacionan con sus miembros y con la sociedad en la que se desempeñan, su enfoque, su misión y su visión, entre otros varios factores, van formando su propia cultura organizacional. Rodríguez (2009) señala que la cultura organizacional es un subsistema complejo que funciona dentro de otros dos sistemas también complejos: organización y contexto.

El desafío de la globalización ocasiona que actualmente organizaciones de todos los campos compitan para aportar valor. El valor es la capacidad de satisfacer o rebasar las necesidades de los clientes de manera eficiente. Las compañías deben ofrecer valor a sus clientes, y los países tienen que ofrecer valor como emplazamientos de negocios.

En la actualidad, esto resulta tan válido para un hospital que ofrece atención sanitaria, como para una fundación que realiza contribuciones benéficas o para una empresa que produce un producto o un servicio. Ofrecer valor social - altos beneficios sociales por dólar invertido - se está convirtiendo rápidamente en el imperativo para cualquier organización que busque fomentar el bien público. (Porter, 2009); (Cantillo, E. \& Escorcia, J., 2011)

Orientando. Temas de Asia Oriental. Sociedad, Cultura y Economía. Revista editada por el Centro de Estudios China-Veracruz de la Universidad Veracruzana, México (Centro de Estudios APEC) / año 10 / número 19/ 
La cultura organizacional será fundamental a la hora de que todos los miembros de la organización tomen decisiones, por otra parte, esta cultura contendrá valores éticos básicos que todos sus miembros deberán compartir entre sí.

En tanto, Robbins y Judgey (2009) destacan siete características de la cultura organizacional y señalan su importancia para medir el rendimiento individual y colectivo en la empresa: innovación, orientación a los resultados, orientación a las personas, orientación a los equipos, minuciosidad, agresividad y estabilidad.

Figura 2. Características de la cultura organizacional

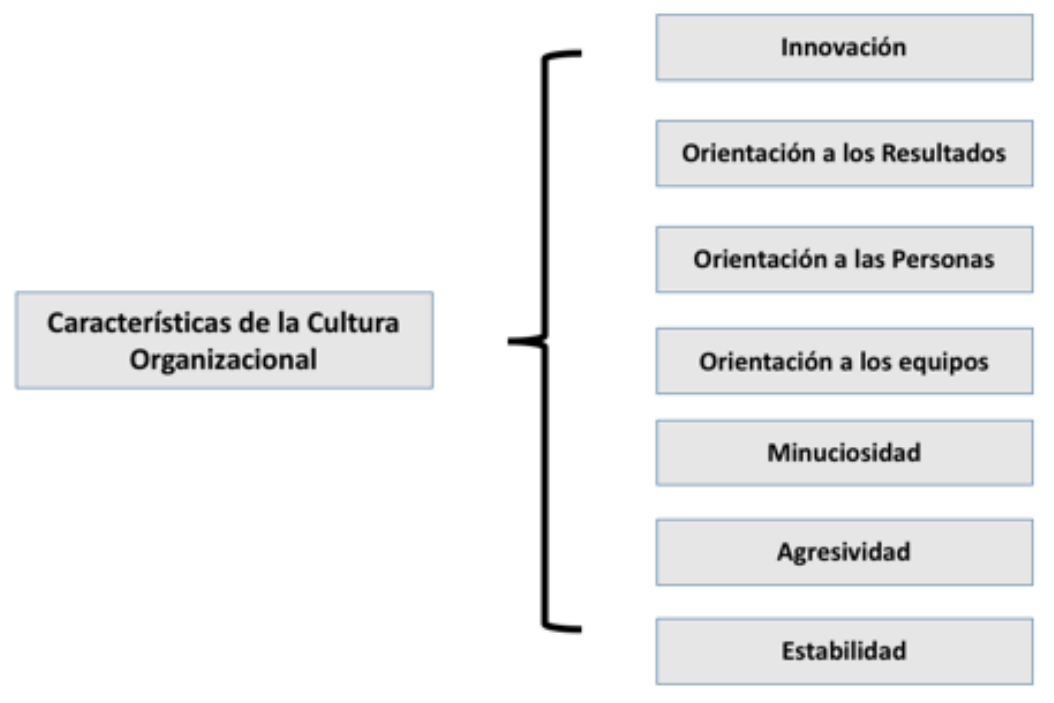

Fuente: Elaboración propia con base en Robbins y Judgey (2009)

\subsection{Cultura nacional modelo de Hofstede-América Latina y China}

América Latina y China han intensificado el intercambio comercial en los últimos 20 años, lo que ha generado también mayor cooperación entre gobierno, empresas y diversas instituciones de ambas regiones; esto ha intensificado el intercambio de personas perteneciente a distintas culturas nacionales. Si bien la cultura nacional es solo una de las variables que pueden influir sobre el comportamiento de las personas en las organizaciones, la misma puede influir como un factor determinante a la hora de tomar decisiones; entendiendo la cultura como una programación colectiva de la mente que se manifiesta de diferentes formas (ver Figura 3). 
Según Hofstede, de entre los múltiples términos que se emplean para describir las manifestaciones de la cultura, hay cuatro que cubren todo el concepto de una manera bastante acertada: símbolos, héroes, rituales y valores. Nos podemos imaginar que son las capas de una cebolla: los símbolos representan la capa más superficial de la cultura y los valores la más profunda, quedando los héroes y los rituales en medio de las dos.

\section{Figura 3. "Onion Diagram” de Hofstede}

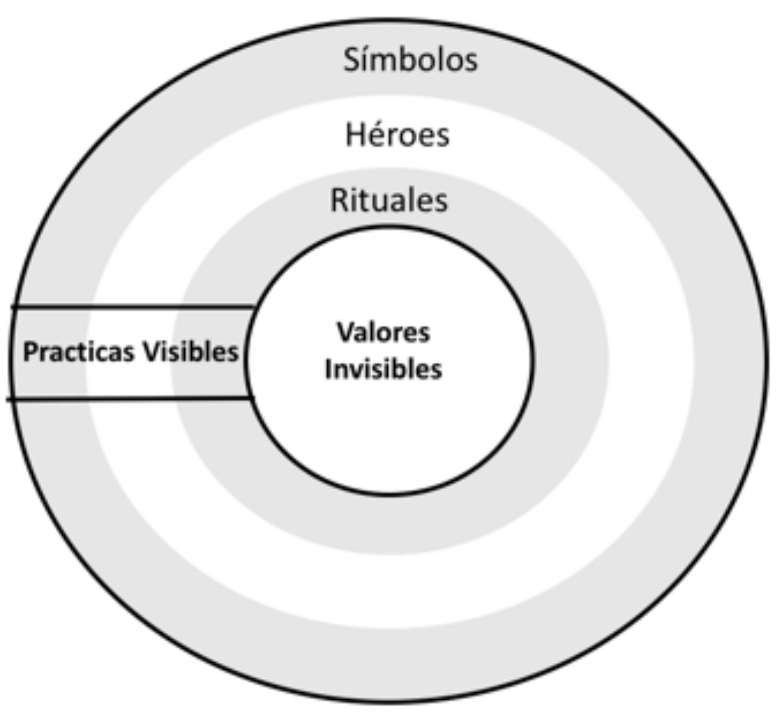

Fuente: Elaborado con base en Hofstede (2001).

En la cultura se pueden distinguir distintas dimensiones, algunas de las cuales poseen ciertos grados de similitud entre países y otras se manifiestan de manera totalmente diferente. Esto hace que se generen diferentes tipos de influencia sobre los individuos de distintas culturas que forman parte de las organizaciones; lograr comprender estas diferencias a la hora de planificar, ejecutar y evaluar, resulta fundamental para ejercer el liderazgo de manera efectiva y poder completar las metas planificadas.

A finales de la década de 1960, Hofstede evaluó los resultados de un estudio internacional realizado por personal del IBM de habla inglesa, generando la idea de dimensiones culturales. Este modelo se fue perfeccionando a lo largo de los años y actualmente sigue siendo una pauta de gran interés para señalar las posibles diferencias entre culturas.

Orientando. Temas de Asia Oriental. Sociedad, Cultura y Economía. Revista editada por el Centro de Estudios 
En este modelo existen seis dimensiones culturales que se pueden evaluar con medidas de 1 a 100:
a) Distancia de poder
b) Individualismo
c) Masculinidad $/$ Feminidad
d) La evasión de la incertidumbre
e) Orientación a largo plazo
f) Indulgencia

\subsubsection{Distancia de poder}

Es la medida en que los miembros menos poderosos de las organizaciones e instituciones, así como de la familia, aceptan y esperan que el poder se distribuya de manera desigual. Argentina tiene una dimensión cultural de distancia al poder relativamente baja, mientras que en China y México ambas son altas; está dimensión tiene gran influencia en la relación de los subordinados con los líderes.

\subsubsection{Individualismo}

El individualismo es la medida en que las personas se sienten independientes, en lugar de ser interdependientes como miembros de un todo más grande. En este sentido, una cultura nacional con 100 puntos implicaría que posee el grado máximo de individualismo; un factor determinante a la hora de actuar en la toma de decisiones.

\subsubsection{Masculinidad/Feminidad}

Esta dimensión se refiere a la distribución de los roles entre hombres y mujeres. En esta dimensión encontramos similitudes entre la cultura nacional de China y la de México.

\subsubsection{La evasión de la incertidumbre}

Se relaciona con la tolerancia de la sociedad a la incertidumbre y la ambigüedad. En el caso de que esta dimensión sea baja, implica que la sociedad está dispuesta a aceptar cambios más fácilmente que en sociedades donde su rango es elevado.

\subsubsection{Orientación a largo plazo}


En una cultura orientada a largo tiempo, la noción básica sobre el mundo es que está en constante cambio, y siempre es necesario prepararse para el futuro. En una cultura orientada a corto tiempo, el mundo es esencialmente como fue creado, de modo que el pasado proporciona una brújula moral y adherirse a él es moralmente bueno. China figura con una dimensión cultural de orientación a largo plazo alta, lo que le permite realizar planificaciones a periodos más largos.

\subsubsection{Indulgencia}

Esta dimensión se ha incorporado a las 5 que originalmente tenía el modelo de Hofstede. Es bueno ser libre en las culturas indulgentes: hacer lo que tus impulsos quieren que hagas, es bueno; los amigos son importantes y la vida tiene sentido. En una cultura restringida, la sensación es que la vida es dura, y el deber, no la libertad, es el estado normal del ser. (Mena, 2018) Con este modelo podemos visualizar que los países de América Latina entre sí y con respecto a China poseen similitudes y diferencias en sus dimensiones culturales.

\section{Liderazgo}

El concepto de liderazgo, así como la forma en que responden los subordinados a determinados estímulos puede variar a través de diferentes culturas. Un líder organizacional deberá orientar los procesos para que se lleven a cabo de manera consecuente y exitosa.

Torres, F. V. C., \& Ríos, G. A. C. (2013) definen que el liderazgo es un proceso relacional más que un ejercicio centrado en el líder como sujeto, en el que el liderazgo emerge a partir de la interacción entre los agentes del sistema. Asimismo, cuando se observa el liderazgo desde el o los líderes -individuos con mayor poder de influencia-se entiende que el poder no hace al líder, pero que sin poder no hay liderazgo; por ello, estudiar este concepto es fundamental.

El líder se retroalimenta del ambiente organizacional y a su vez debe generar cambios en el mismo. Por tanto, el liderazgo efectivo puede influir el desempeño exitoso de las organizaciones. Pero el liderazgo es un proceso de aprendizaje no es estático. En la

Orientando. Temas de Asia Oriental. Sociedad, Cultura y Economía. Revista editada por el Centro de Estudios China-Veracruz de la Universidad Veracruzana, México (Centro de Estudios APEC) / año 10 / número 19/ 
actualidad, los grandes cambios que se están produciendo a nivel tecnológico y la internacionalización acelerada de las organizaciones, deberá ser un factor que los líderes tendrán que asimilar en beneficio del desarrollo de las organizaciones y de sus miembros.

\subsection{Cultura nacional y liderazgo}

Uno de los problemas principales al analizar diferencias culturales es la dificultad de estandarizar las culturas nacionales. Para Molero, F (2002) asumir que los habitantes de una determinada nación (es decir, las personas que tienen el mismo pasaporte) comparten una misma cultura es cuestionable porque dentro de una misma nación pueden existir grupos sociales, étnicos o religiosos con subculturas diferentes.

Al hacer una investigación transcultural (cross-cultural), debemos tener una base teórica para poder realizar modelos y asumir hipótesis para realizar conclusiones y analizar situaciones determinadas. De esa manera, en las investigaciones se ha tendido por lo general a equiparar "nación” y "cultura”, debido, por una parte, a que los habitantes de una nación suelen estar expuestos a una serie de estímulos comunes (lenguaje, medios de comunicación, sistema educativo y político, etc.) y, por otra parte, a que la mayoría de las estadísticas sociodemográficas reflejan datos nacionales; esto ha hecho más fáciles las investigaciones que toman la nación como unidad de análisis. Probablemente por estas razones Hofstede considera la nación como unidad de análisis en su investigación transcultural. (Molero, F, 2002)

Sin embargo, si bien son muy diversos los aspectos que se debe tener en cuenta cuando se lidera una organización, la cultura nacional es un aspecto fundamental a considerar a la hora de ejercer un liderazgo que promueva integración, desarrollo e innovación entre los miembros de la organización, en beneficio del cumplimiento de los objetivos.

\subsection{Liderazgo intercultural}

El liderazgo en las organizaciones se enfrenta a un cambiante escenario en donde la interculturalidad comienza a tener un rol fundamental para el cumplimiento de los objetivos y el desarrollo de las organizaciones en un contexto de gran globalización. Por ello ha sido frecuente observar los problemas de adaptación, tanto de los empleados a 
prácticas organizacionales oriundas de otros contextos culturales, como de los gerentes provenientes de otras culturas a las prácticas locales.

Resulta fundamental que en las organizaciones los líderes puedan comprender este tipo de situaciones y promover un tipo de liderazgo intercultural. Para eso es importante entender la cultura de los empleados en la organización, porque la cultura nacional de un empleado puede influir en su percepción del ambiente de trabajo y sus componentes.

El líder organizacional debe tener una gran comprensión de los subordinados para poder generar un ambiente en dicha organización que promueva el desarrollo y la innovación. Según Kuchinke (1999), para que los líderes sean capaces de realizar las expectativas de los subordinados, su comportamiento de liderazgo debe estar dentro del contexto cultural particular, de lo contrario, los líderes no serán eficaces.

Las culturas personales y nacionales se derivan de la gente y los valores personales que impregnan el papel del liderazgo con gestión eficaz y actuaciones exitosas. (Byrne \& Bradley, 2007) Una de las características más importantes de los líderes es tener una idea con la que pueden ver lo que ocurre en el grupo, organización o sociedad y diagnosticar la forma en que se podría resolver.

Los líderes y los futuros líderes organizacionales deben hacer frente a circunstancias complejas, cambiantes y difíciles de explicar. Los administradores de las empresas internacionales se enfrentan más que nunca a una situación internacional intensa y en rápida evolución. La tendencia de la aldea económica mundial es evidente, probablemente en el siglo XXI, bien conocido como el siglo del "mundo global". (McFarland, senen, y Childress, 1993)

Habida cuenta de que un liderazgo institucional eficaz es fundamental para el éxito de las operaciones internacionales, la globalización de la organización industrial plantea muchos problemas de organización y liderazgo. Por ejemplo, en las organizaciones transnacionales de todo el mundo, la diversidad cultural del personal plantea importantes problemas para el diseño y la dirección de las organizaciones transnacionales. (House, R. J., \& Mansor, N.1999)

Orientando. Temas de Asia Oriental. Sociedad, Cultura y Economía. Revista editada por el Centro de Estudios China-Veracruz de la Universidad Veracruzana, México (Centro de Estudios APEC) / año 10 / número 19/ 
Este tipo de liderazgo deberá analizar la administración de las organizaciones desde una perspectiva multicultural. Esto significa la capacidad de dichos líderes para tomar la perspectiva del otro dentro del contexto cultural, comprender diferencias culturales y adaptarse rápidamente cuando se encuentre con individuos o grupos que pertenecen a otras culturas.

Gelfand, Erez y Aycan (2007) interpretan los comportamientos generales que un líder intercultural debe incorporar como comportamiento organizacional intercultural. Esto se define como "similitudes y diferencias interculturales en los procesos y comportamientos en el trabajo, y la dinámica de las interfaces transculturales en contextos nacionales e internacionales multiculturales".

En el caso específico de las empresas, un liderazgo empresarial intercultural permitirá apreciar las diferentes dimensiones culturales de los miembros de la organización pertenecientes a diferentes culturas; así como planificar y tomar decisiones que generen mayor satisfacción laboral y compromiso organizacional por parte de los subordinados. Como se aprecia en la Figura 4, el liderazgo intercultural aplicado puede tener un resultado positivo en la actitud de los empleados.

\section{Figura 4. Liderazgo intercultural e intercambio entre empresas}

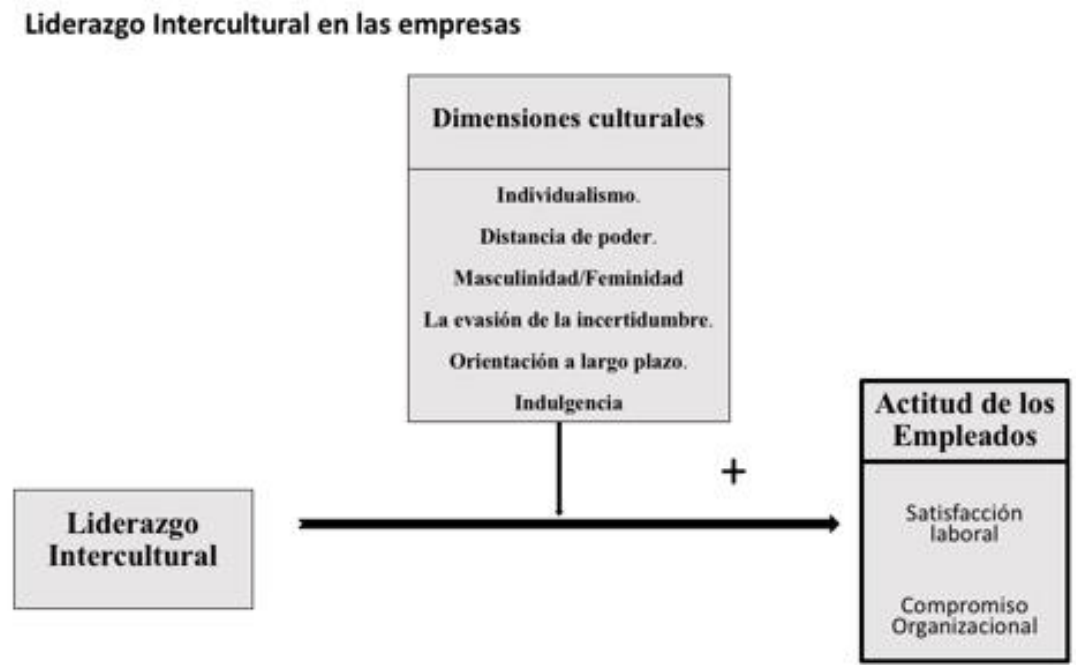

Fuente: Elaboración propia.

\subsection{Interculturalidad y comunicación intercultural}


El manejo adecuado de la gestión intercultural puede generar a su vez la innovación, ya que el análisis de situaciones desde distintos puntos de vista puede generar que la interacción de miembros de diferentes culturas enriquezca el intercambio y promueva la innovación.

Un punto fundamental de la gestión intercultural es la comunicación intercultural, ya que distintas percepciones pueden generar problemas de comunicación; la comunicación intercultural es aquella comunicación de forma interpersonal donde intervienen personas con unos referentes culturales lo suficientemente diferentes como para que ello pueda suponer una barrera importante que altere la eficacia comunicativa. (Baños, R. 2012)

En este punto cabe señalar la importancia de la eficacia comunicativa. Esta no implica una comunicación perfecta controlada y exenta de ambigüedades, sino que se entiende como el grado de comprensión aceptable o suficiente entre las personas en contacto. (Rodrigo, 1999) De esta forma, solventar las posibles barreras tanto personales como contextuales que puede suponer un encuentro multicultural, es de esencial para alcanzar dicha eficacia. (Baños, R. 2012)

Rizo, M., \& Romeu, V. (2006) concluyen que la interculturalidad es, antes que cualquier otra cosa, una postura. Una postura híbrida, una tarea por hacer, una tarea inconclusa que plantea la necesidad de buscar caminos para la integración, la armonía y el desarrollo humano. Por ello, el ser intercultural se corresponde fundamentalmente no con la ejecución concreta y particular de estrategias o acciones encaminadas a tal fin, sino con el acto mismo de pensar y actuar conforme a un pensamiento intercultural.

El pensamiento intercultural es fundamental para cualquier tipo de intercambio relacionado con la Franja y la Ruta, para poder lograr reducir la brecha entre la teoría y la práctica, citada anteriormente. El hacer y el saber intercultural nos coloca en el punto medio de los ámbitos de tensión entre lo ideal y lo real, entre el fin perseguido y la realidad que lo produce y lo acoge. Su falta de concreción no comporta necesariamente inoperancia, si no más bien la clara convicción de que se trata de un camino en constante construcción, cuyo recorrido es en la misma medida fruto de un pensar y un hacer intercultural. (Rizo, M., \& Romeu, V. 2006)

Orientando. Temas de Asia Oriental. Sociedad, Cultura y Economía. Revista editada por el Centro de Estudios China-Veracruz de la Universidad Veracruzana, México (Centro de Estudios APEC) / año 10 / número 19/ 
Esta postura intercultural es parte de un proceso individual que permite a los líderes dirigir organizaciones formadas por miembros de distintas culturas, a favor de lograr los objetivos de la organización.

Figura 5. Influencia del intercambio de personas bajo el liderazgo intercultural efectivo

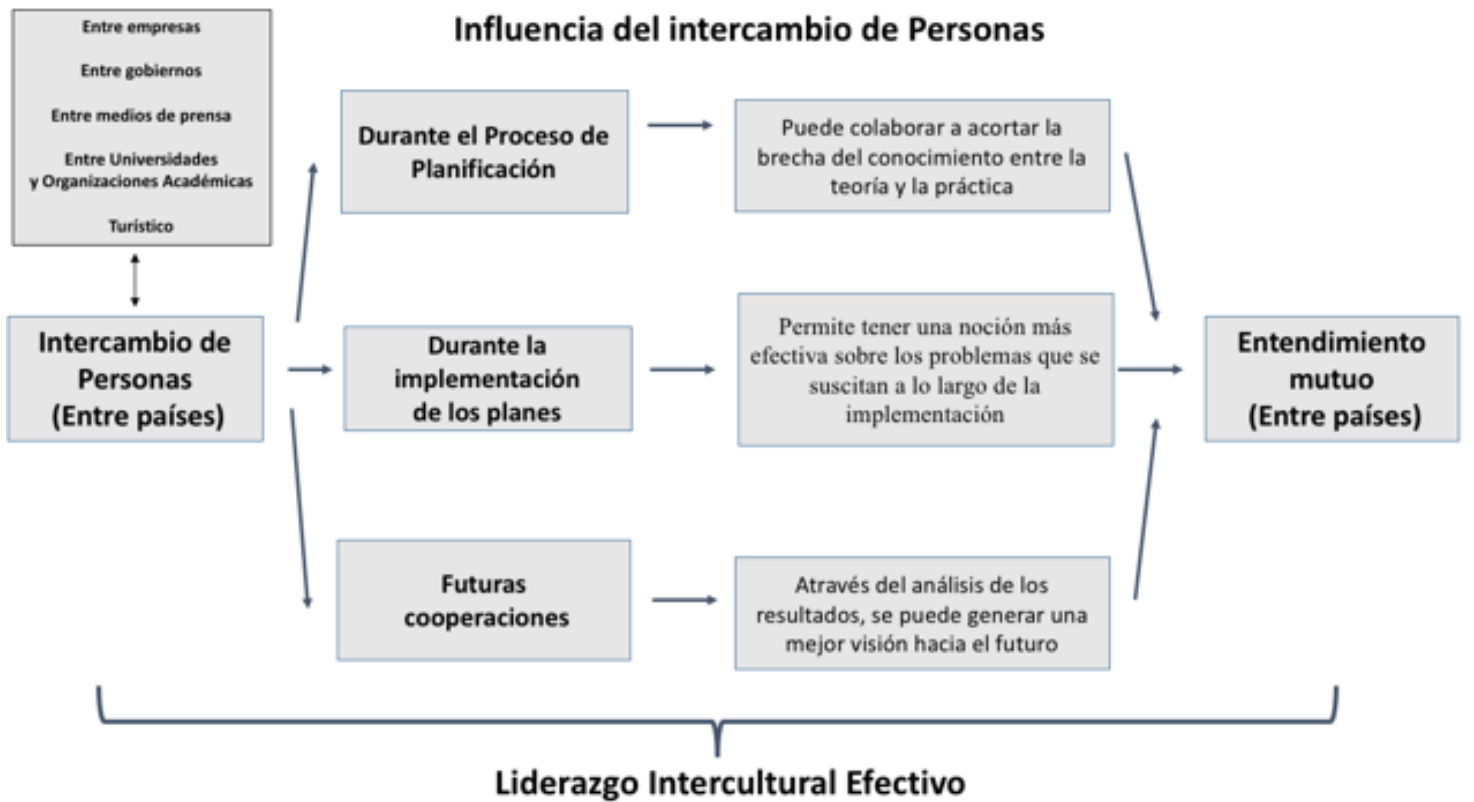

Fuente: Elaboración propia.

\section{Conclusiones}

El mundo globalizado ha generado cambios en las estructuras organizacionales de los países. La iniciativa de la Franja y la Ruta, propuesta en 2013, integra el deseo de una comunidad de destino compartido; en los últimos dos años, gran parte de los países de América Latina se han ido incorporando a dicha iniciativa, lo que ha generado cada vez más proyectos conjuntos y un creciente intercambio de personas.

Tal progreso exige a las organizaciones mayor comprensión de los factores de la cultura nacional, que influyen en las decisiones de sus miembros. El intercambio de personas se ha generado en diversos ámbitos, entre empresas, entre gobiernos, entre medios de prensa, entre universidades y centros de investigación y también a nivel turístico.

En este sentido, se deberán comprender las distintas dimensiones de las culturas nacionales de América Latina y China y su influencia en las prácticas organizacionales. Para ello resulta fundamental el rol del liderazgo: los líderes deberán tener una visión 
cross-cultural y multidimensional a la hora de planificar, tomar decisiones y evaluar los resultados obtenidos en las organizaciones que dirigen. Esto permitirá acelerar el entendimiento mutuo entre naciones.

Si bien la cultura nacional resulta muy compleja para estandarizar, utilizando el modelo de dimensiones culturales de Hofstede y ciertas teorías sobre liderazgo intercultural, se deberán materializar nuevas formas de administración que permitan mayor entendimiento cultural; permitiendo así que los proyectos transnacionales se desarrollen de manera activa y efectiva. Por tanto, es muy importante la comunicación intercultural para poder aprovechar la experiencia de los intercambios internacionales como una base fundamental para el entendimiento mutuo en la búsqueda de una comunidad de destino compartido.

\section{Bibliografía}

Baños, R. (2012). No Verbales en la Comunicación Intercultural. Ra-Ximhai, 8(2).

Beebe, Beebe, \& Redmond. (2005). Building our understanding: Culture insights communicating with Hispanic/Latinos. CiteSeerX Scientific Literature Digital Library and Search Engine.

Byrne, G. J., \& Bradley, F. (2007). “Culture's influence on leadership efficiency: How personal and national cultures affect leadership style". In Journal of Business Research, 60(2), pp. 168-175.

Cantillo, E. \& Julio, E. (2011). Influencia de la cultura organizacional en la competitividad de las empresas.

Centro de Estudios Superiores en Turismo. (1997). El turismo cultural en México. Resumen ejecutivo del estudio estratégico de viabilidad del turismo cultural en México. México: Secretaría de Turismo.

Gelfand, M. J., Erez, M., \& Aycan, Z. (2007). "Cross-cultural organizational behavior”. In Annu. Rev. Psychol., 58, 479-514.

Hofstede, G. (1980). Culture's consequences: International differences in work-related values. London: Sage.

Orientando. Temas de Asia Oriental. Sociedad, Cultura y Economía. Revista editada por el Centro de Estudios 
Hofstede, G. (2001). Culture's consequences: Comparing values, behaviors, institutions and organizations across nations. Thousand Oaks, CA: Sag

House, R. J., Wright, N. S., \& Aditya, R. N. (1997). "Cross-cultural research on organizational leadership: A critical analysis and a proposed theory". In P. C. Earley \& M. Erez (Eds.). New Perspectives in International Industrial Organizational Psychology. San Francisco: New Lexington, pp. 535-625.

House, R. J., \& Mansor, N. (1999). Cultural Influences on Leadership and Organizations: Project Globe (Vol. 1). Advances in Global Leadership.

Kuchinke, K. P. (1999). "Leadership and culture: Work-related values and leadership styles among one company's US and German telecommunication employees". In Human resource development quarterly, 10(2), 135-154.

Mena, Cecilia (2018). “El modelo de seis dimensiones de cultura”. En Forbes. México https://www.forbes.com.mx/el-modelo-de-seis-dimensiones-de-la-cultura/

McFarland, L. J., Senen, S., \& Childress, J. R. (1993). Twenty-first-century leadership. New York: Leadership Press.

Molero, F. (2002). "Cultura y liderazgo. Una relación multifacética”. En Boletín de Psicología, 76, pp. 53-75.

Omar, A., \& Urteaga, A. F. (2010). El impacto de la cultura nacional sobre la cultura organizacional. Universitas Psychologica, 9(1), pp. 79-92.

Porter, M. E. (2009). Ser Competitivo. Barcelona: Deusto.

Rizo, M., \& Romeu, V. (2006). "Cultura y comunicación intercultural. Aproximaciones conceptuales”. In e-Compós (Vol. 6).

Robbins, S. P. y Judgey, T. A. (2009). Comportamiento organizacional (Décimo tercera ed.). México: Mc Graw Hill.

Rodrigo, M. (1999). La comunicación intercultural. Barcelona: Anthropos.

Smith, P.B.-Bond,M.H.(1998): Social Psychology across cultures (2a edición). Londres: Prentice Hall.

Smith, G. (1966). Communication and culture. New York: Holt, Rinehart and Winston.

Smith, P.B., Dugan, S. Peterson\& M.F. Leung, K. (1998). "Individualism/collectivism and the handling of disagreement". In A 23 Country study, International Journal of Inter-cultural Relationships, 22, (3), pp. 351-367. 
Triandis, H.C.,(1994): "Cross-cultural Industrial and Organizational Psychology". En: Triandis, H.C. Dunette, M.D \& Hough, L.M. (Eds.) Handbook of Industrial and Organizational Psychology (2a ed.). Palo Alto: Consulting Psychologist Press.

Triandis, H. C. (1995). Individualism and Collectivism. Boulder. CO: Westview Press.

Torres, F. V. C., \& Ríos, G. A. C. (2013). "Liderazgo, poder y movilización organizacional”. En Estudios gerenciales, 29(126), 72-76.

Trujillo Sáez, F. (2005). En torno a la interculturalidad: reflexiones sobre cultura y comunicación para la didáctica de la lengua.

Wang Yiwei. (2019). "La connotación teórica de La Franja y la Ruta y su importancia para América Latina". En Guo Cunhai y Mera, C. (Eds.). La Franja y la Ruta y América Latina: Nuevas oportunidades y desafíos. China: China Intercontinental Press.

Williams, R. (1983). Keywords. London: Fontana.

Zottele, E. (2015). "Liderazgo paternalista: Un punto de contacto entre China y México". En Orientando Temas de Asia Oriental, Sociedad, Cultura y Economía, (9).

Zottele, E. \& Qian, W. (2017). "La Franja y la Ruta: Oportunidad para América Latina y búsqueda de un desarrollo sostenible". En Orientando Temas de Asia Oriental, Sociedad, Cultura y Economía, (13).

Orientando. Temas de Asia Oriental. Sociedad, Cultura y Economía. Revista editada por el Centro de Estudios China-Veracruz de la Universidad Veracruzana, México (Centro de Estudios APEC) / año 10 / número 19/ 